\title{
Preface - SpS8 - The Galactic Plane
}

\author{
N. A. Walton, A. Damineli, co-chairs SOC, M. G. Hoare, J. E. Drew, editors
}

The first digital astronomical surveys emphasised exploration of the sky away from the crowded Galactic Plane. But now, increased computing power has made it possible to take on comprehensive surveying of the Galactic Plane even at high spatial resolution and down to faint magnitude limits. A number of ambitious wide-area surveys sampling high energies, optical wavelengths, the infrared, sub-millimetre and radio ranges are complete, in process, or about to begin. The goals of these surveys are as broad as Galactic science itself, but are mainly focused either on solving key problems in star formation and stellar evolution, or on mapping the complex substructures of the Galactic bulge and disk in order to see more clearly how the whole is constructed. This meeting brought together researchers directly involved in the many surveys, along with specialists in the observations and modelling of the ISM, stellar evolution, and the structure of the Galactic Disk and Bulge.

The programme of this Special Session, spread over 4 days, was grouped into ten topics. These were: 1 . The new generation surveys; 2. Dust in the Milky Way; 3. Structure in the Galaxy; 4. Chemical picture of the Galaxy; 5. Disentangling the star formation process; 6. Mapping star formation in the Galactic disk; 7. Multi-object multi-wavelength galactic plane studies in the VO era; 8. End-states of stellar evolution; 9. Luminous evolved stars; 10. Perspective on the future.

The major threads running through the meeting were the preoccupations with identifying and locating structures within the Galactic Plane (disk and bulge), and what we are learning about its stellar populations and the star-forming process. The discussion of the structure did draw on the entire wavelength range from the high energies sampled by HESS up to the HI $21 \mathrm{~cm}$ line work of GALFA. We heard about the progress being made in 3-D extinction mapping at optical and NIR wavelengths, and about the ongoing debate regarding the character of the disk's spiral arm structure and the bar in the bulge. The two stellar arm picture versus the four gaseous, star-forming arms was a recurrent theme throughout the meeting. Methanol masers were discussed both as phenomena linked to massive star formation and as targets for VLBI distance determinations. We also heard about the crucial matter of chemical abundance determinations from both stars and nebulae, based on optical data. The significant body of work stimulated by the Spitzer/GLIMPSE survey was very much in evidence during the meeting, as was the growing input from IPHAS. The future was anticipated in talks on LSST and Pan-STARRS.

It should go down on the record that Andrew Walsh wowed the audience with a magnificent sung (and versified) poster pitch. And it should also be mentioned that Anne Green won the poster competition and a bottle of cachaça with a high impact presentation on 'The southern sky at $843 \mathrm{MHz}$ '. Specific thanks are due to SOC member, Gene Magnier for his enthusiastic help in the judging of the posters, alongside Nic and Janet. Last but not least, it is a great pleasure to acknowledge the financial support underpinning the Rio General Assembly as a whole, the efforts of the GA's LOC that made the venue work, and the active support of the members of the wider SOC in designing the programme for this interesting meeting. 